\title{
VCF2PopTree: a client-side software to construct population phylogeny from genome-wide SNPs
}

\author{
Sankar Subramanian ${ }^{\text {Corresp., } 1}{ }^{1}$ Umayal Ramasamy ${ }^{1}$, David Chen ${ }^{2}$ \\ ${ }^{1}$ GeneCology Research Centre, The University of the Sunshine Coast, Sippy Downs, QLD, Australia \\ 2 School of Information and Communication Technology, Griffith University, Nathan, QLD, Australia \\ Corresponding Author: Sankar Subramanian \\ Email address: ssankara@usc.edu.au
}

In the past decades a number of software programs have been developed to infer phylogenetic relationships between populations. However, most of these programs typically use alignments of sequences from genes to build phylogeny. Recently, many standalone or web applications have been developed to handle large-scale whole genome data, but they are either computationally intensive, dependent on third party software or required significant time and resource of a web server. In the post-genomic era, researchers are able to obtain bioinformatically processed high-quality publication-ready whole genome data for many individuals in a population from next generation sequencing companies due to the reduction in the cost of sequencing and analysis. Such genotype data is typically presented in the Variant Call Format (VCF) and there is no simple software available that directly uses this data format to construct the phylogeny of populations in a short time. To address this limitation, we have developed a user-friendly software, VCF2PopTree that uses genome-wide SNPs to construct and display phylogenetic trees in seconds to minutes. For example, it reads a VCF file containing 4 million SNPs and draws a tree in less than 30 seconds. VCF2PopTree accepts genotype data from a local machine, constructs a tree using UPGMA and Neighbour-Joining algorithms and displays it on a webbrowser. It also produces pairwise-diversity matrix in MEGA and PHYLIP file formats as well as trees in the Newick format which could be directly used by other popular phylogenetic software programs. The software including the source code, a test VCF file and a documentation are available at: https://github.com/sansubs/vcf2pop.[b] [b] 
2

3 VCF2PopTree: a client-side software to construct population phylogeny from genome-wide SNPs

\author{
Sankar Subramanian ${ }^{1 *}$, Umayal Ramasamy ${ }^{1,2}$ and David Chen ${ }^{2}$ \\ ${ }^{1}$ Gene Cology Research Centre, The University of the Sunshine Coast, 90 Sippy Downs Drive, Sippy \\ Downs Qld 4556, Australia \\ ${ }^{2}$ School of Information and Communication Technology, Griffith University, 170 Kessels Road, Nathan, \\ Qld 4111, Australia
}

${ }^{*}$ Corresponding author

Address for correspondence:

GeneCology Research Centre

University of the Sunshine Coast

90 Sippy Downs Drive

Sippy Downs QLD 4556

Australia

Phone: + 61-7-5430 2873

Fax: +61-7- 54302881

E-mail: ssankara@usc.edu.au

5 Running head: VCF2PopTree

Keywords: VCF, Phylogeny, UPGMA, Neighbour-Joining, MEGA and PHYLIP

6

7

8

9

10

Peer] reviewing PDF | (2019:04:37132:2:0:NEW 4 Nov 2019) 


\section{Abstract}

In the past decades a number of software programs have been developed to infer phylogenetic relationships between populations. However, most of these programs typically use alignments of sequences from genes to build phylogeny. Recently, many standalone or web applications have been developed to handle large-scale whole genome data, but they are either computationally intensive, dependent on third party software or required significant time and resource of a web server. In the post-genomic era, researchers are able to obtain bioinformatically processed high-quality publication-ready whole genome data for many individuals in a population from next generation sequencing companies due to the reduction in the cost of sequencing and analysis. Such genotype data is typically presented in the Variant Call Format (VCF) and there is no simple software available that directly uses this data format to construct the phylogeny of populations in a short time. To address this limitation, we have developed a user-friendly software, VCF2PopTree that uses genome-wide SNPs to construct and display phylogenetic trees in seconds to minutes. For example, it reads a VCF file containing 4 million SNPs and draws a tree in less than 30 seconds. VCF2PopTree accepts genotype data from a local machine, constructs a tree using UPGMA and NeighbourJoining algorithms and displays it on a web-browser. It also produces pairwise-diversity matrix in MEGA and PHYLIP file formats as well as trees in the Newick format which could be directly used by other popular phylogenetic software programs. The software including the source code, a test VCF file and a documentation are available at: https://github.com/sansubs/vcf2pop. 
34

35

36

37

\section{Introduction}

One of the major tasks in genetics and evolutionary biology is to infer the ancestral relationship between populations and species. For this purpose, a number of mathematical and statistical algorithms have been developed. To implement these algorithms, computationally efficient software programs were developed. However, these software such as MEGA (Kumar et al. 2016), PHYLIP (Felsenstein 2005), PAUP (Wilgenbusch \& Swofford 2003) and BEAST (Drummond et al. 2012) are suited only for gene-based sequence data. In the recent past, a series of programs such as RaxML (Stamatakis 2006), ExaML (Kozlov et al. 2015) and MP-EST (Liu et al. 2010) have been developed to infer phylogenetic relationship using whole genome data. A number of sophisticated tree-building software such as TreeMix (Pickrell \& Pritchard 2012) and QPgraph (Patterson et al. 2012) have also been developed to accommodate the number of potential admixture events while inferring the phylogeny. However, to use these software programs, the genome data need to be processed in specific formats such as alignments or allele frequencies.

With the advent of the next generation sequence techniques, large-scale whole genome data containing millions of Single Nucleotide Variations (SNVs) are generated for populations. The whole genome data is typically presented in the Variant Call Format (VCF) and there was a need for genetic software to construct population phylogeny that directly use this data format. To address this limitation a number of software programs have been developed in the recent past. However, these programs are either computationally intensive, time consuming, heavily dependent on third party software or require significant time and resource of a web server. 
56 The software programs that reads VCF data are either standalone or web server-based applications.

57 While some of the standalone programs such as plink (Pickrell \& Pritchard 2012), ngsDist (Vieiran

58

59

60

61

62

63

64

65

66

67

et al. 2016) and VCF2Dis (https://github.com/BGI-shenzhen/VCF2Dis) estimate pairwise distance matrix from VCF files, others such as FastMe (Lefort et al. 2015) and MEGA (Kumar et al. 2016) infer the phylogeny using the matrix. Hence there was a need for software that read VCF files and draw phylogenetic tree directly. To accomplish this a handful of standalone applications such as SNPhylo (Lee et al. 2014), VCF-Kit (Cook \& Andersen 2017), and VCFtoTree (Xu et al. 2017) have been developed. However, these software pipelines need to be installed in a local computer. Furthermore, these programs are dependent on a series of other software such as $b w a$ (Li \& Durbin 2009), samtools (Li et al. 2009) and/or MUSCLE (Edgar 2004). Therefore, an adequate level of computer expertise is required to implement and run the standalone programs. On the other hand, web server-based programs such as SNiPlay (Dereeper et al. 2015), and CSI Phylogeny (Kaas et al. 2014) take significant amount of time to produce a tree using the data from a VCF file. This is partly due to the time taken to upload the large-data set to a server from the user's local machine, which depends on the web traffic and internet speed. Furthermore, both standalone and serverbased applications perform a series of data processing steps through software pipelines, which also cause significant time delay.

Due to the reduction in the cost of sequencing and bioinformatic analysis, it is now possible to obtain processed whole genome data for many individuals. Using standard bioinformatic data processing pipelines most of the sequencing service providers deliver high quality publicationready genotype data for whole genomes in the form of VCF files. Hence population geneticists now need a simple program that reads this data in VCF files and construct a phylogenetic tree in a 
79 short time as there is no need of any data processing routines. Therefore, the current study is aimed

80 to the address this important limitation in genomic research. Hence, we developed a JavaScript

81 based client-side software to infer phylogenetic relationship using genome-wide SNV data.

\section{Methods}

\section{Implementation}

The software, VCF2PopTree was written in JavaScript, which runs purely within the user's computer/browser. This program reads VCF files including compressed (gzipped) files. A VCF

87 file contains genotype information in the form of ' 0 's and ' 1 's to denote reference and alternate

88 alleles. VCF2PopTree is designed to read and process the input data line-by-line so it is able to

89 handle large data files without running out of memory. The program considers only biallelic SNPs and ignores insertion-deletions (Indels) and SNVs with missing information (./.). Furthermore, based on the user's thresholds (entered in the textboxes) for quality scores and coverage depths, the program filters SNVs. Using the genotype data, two types of measures namely, genetic and

93 drift distances are calculated. To compute pairwise genetic distance between two diploid genomes

94 four pairwise comparisons are performed, and the average is estimated. For instance, the genetic distance for heterozygous SNVs from two genomes $(0 / 1$ and $0 / 1)$ is $0.5(2 / 4)$. For estimating drift

96 distance, only the dissimilarity of the allele frequencies is considered and for the above-mentioned

97 comparison the drift distance is 0 as the allele frequencies are the same. The distance estimates

98 obtained for each site or SNV are summed to get the total number of differences for the whole genome. The pairwise matrix of these differences are directly used to construct a phylogeny. 
100 To handle missing data there are two options provided. By selecting Use SNVs present in all 101 genomes the program will use only the SNVs (passing the threshold score and coverage) that are

102 present in all genomes. In contrast, selection of the alternative option, Use SNVs for each pair of 103 genomes will result in including all SNVs that pass through the filters and are present in at least 104 one the pair of genomes. If the total genome length is provided, the program converts the 105 differences to proportions of differences ( $p$-distance) and a Jukes-Cantor correction is also 106 implemented. In this analysis numbers of SNVs with missing information (./.), filtered SNVs 107 (based on user's threshold values for quality scores and depth of coverage) and SNVs with more 108 than two alleles are subtracted from the total genome length. The pairwise divergence matrix is 109 then used to infer the phylogenetic relationship using the UPGMA (Sokal \& Michener 1958) and 110 the classical Neighbour-Joining (Saitou \& Nei 1987) algorithms and the resulting tree is presented 111 in the popular Newick or parenthetical format. The Newick formatted phylogeny is used to draw 112 the tree on the browser using the JavaScript package, d3.phylogram.js. Note that the program 113 requires genotype data from at least four genomes in order to build a tree.

\section{Features}

116 The main web page of VCF2PopTree has three major sections (Figure 1A). First section primarily 117 performs file reading and pairwise divergence calculations. VCF2PopTree reads VCF or compressed (gzipped) VCF files. The user has options to filter SNPs based on quality (Phred) scores and depth of coverage. The threshold values have to be entered before loading the input

120 file. If the user changes the threshold values, the input file has to be reloaded again. After the 121 input file is chosen a progress bar is displayed to inform whether the file is being read or the 122 pairwise distance is being calculated, which are the major time-consuming steps. Once the above 
123 steps are completed the progress bar informs the user, who can then choose various options listed

124 in the second section of the program to build and display trees and distance matrices on the third

125 section of the program. The phylogeny could be inferred using all genomes or only a set of selected

126 genomes (at least four) by entering the names in the text area, which appears only if the latter

127 option is selected.

128 As explained in the implementation section, genetic and drift distances could be obtained by

129 choosing the appropriate radio buttons. The pairwise matrices are calculated using the number of 130 differences, $p$-distance or with Jukes-Cantor correction. This is achieved by checking the relevant

131 radio buttons and the genome size has to be provided in the textbox to compute $p$ - and JC distances.

132 The genome size textbox appears only if the options for $p$ - or $J C$ distances are selected. There are 133 two radio buttons to infer phylogenetic relationship between populations using UPGMA and

134 Neighbour-Joining algorithms and the latter method produces an unrooted tree. Two more radio 135 buttons are provided to draw the phylogenetic tree in a rectangular or circular style. Apart from 136 drawing trees VCF2PopTree also produces the tree file in the popular newick format by checking 137 the radio button "Newick format" (Figure 1C). Finally, this program produces pairwise diversity 138 matrix in the popular MEGA (Figure 1B) and PHYLIP formats and the last two radio buttons 139 should be used for this purpose respectively. Once the file is read and pairwise distances are 140 calculated the Draw is activated.

\section{Results}

143 Performance 
144 VCF2PopTree is a simple and straight forward program to use, which requires one click to read 145 the VCF file and compute pairwise distances and another to view the phylogeny of a population. 146 VCF2PopTree is designed to run on personal computers with moderate specifications. To display 147 a phylogenetic tree, it takes a few seconds to minutes depending on the number of SNVs as well 148 as the number of samples/individuals. For example, it takes only 29 seconds to display the 149 phylogeny of 10 individuals based on 4 million SNPs from a VCF file using a Windows computer 150 with 8GB RAM and Intel Core i5 processor. The display time was 3.57 minutes for a VCF file 151 with 100 genomes and 2 million SNPs. VCF2PopTree is compatible with all population browsers 152 including Chrome, Opera, Edge and Firefox and works equally efficient in Mac, Windows and Linux (Ubuntu). Furthermore, it displays the tree in a mobile phone (iPhone and Android) if the 154 input file size is small.

\section{Comparison with Galaxy}

To our knowledge Galaxy (https://usegalaxy.org/) is the only available online software that accepts VCF files from large genomes such as vertebrates and constructs population phylogeny. Hence, we compared the performance of our program with that of Galaxy. The speed of execution depends on two factors, namely the number of genomes and the number of SNPs (or sites) in the VCF file. Therefore, we first compared the performance of the two programs using the number of SNPs. Figure 2A shows a linear increase in the execution times of both software with the number of SNPs and the correlation is highly significant for both comparisons $(r=0.98$ and 0.99 respectively, $P<10^{-6}$ ). However, on an average VCF2PopTree is an order faster than Galaxy in for 400,000 lines, it was only 15 times for 4 million lines. Using the equations of the two lines 
167 revealed that the difference becomes 10-fold and stays the same (reaches an asymptote) after the 168 number of SNPs reaches 100 million and above. The performance based on the number of 169 genomes revealed a highly significant linear and positive relationship for both software $(r=0.989$ 170 and 0.992 respectively, $P<10^{-6}$ ) (Figure 2B). However, VCF2PopTree is at least seven-fold faster 171 than Galaxy and this difference was 17 times higher for 10 genomes and 7.5 times higher when 172 the number of genomes becomes 100. Extrapolations using the linear equations showed that the 173 difference hovers around 7 times even when the number of genomes is 100,000 .

174 Apart from the slow execution time, to use Galaxy, the user has to open a web account and then 175 the VCF file need to be uploaded to the server and converted to gd_snp or gd_genotype format 176 before obtaining a phylogenetic tree. In contrast, only two single clicks are required for 177 VCF2PopTree to read and create a tree on a web browser. Therefore, our program is much more user-friendly and immensely useful for users with limited computer skills. Furthermore, the above-mentioned execution time of Galaxy was also based on the speed of internet connection as well as the waiting times on the queue. In contrast, internet connection is not required for VCF2PopTree as it reads the VCF file from the local machine and there is no issue of queuing in the execution.

Discussion

Since this is a client-side software, VCF2PopTree.html has to be downloaded to the local computer 186 as "Download Zip" from the github server (https://github.com/sansubs/vcf2pop). To examine the

187 functionality of the software we obtained a compressed VCF file (test.vcf.gz) from the Simons 188 Genome Diversity Project containing about half a million SNPs from ten human populations 
189 (Mallick et al. 2016). The input file is read by clicking the choose file button after providing the 190 values for quality and depth of coverage in the appropriate text boxes. Without those values the 191 program considers all SNPs for further analysis. Once the input file is selected a progress bar 192 appears to indicate the status. After the file is read, pairwise distances are calculated and kept in 193 the memory of the program. The user can then select relevant radio buttons, enter the names of 194 the genomes and genome size and click the button Draw. If no names are entered in the text area 195 for genome selection the program will use all genomes. Similarly, if the genome size is not 196 provided phylogeny is inferred based on the number of pairwise differences. The phylogenetic 197 tree or the text area containing the pairwise distance matrix or newick tree format is displayed at 198 the third section of the program beneath the Draw button. The display can be redrawn multiple 199 time by changing different options without reading the input file as the pairwise distance matrix 200 has already been stored in the memory. A number of alert windows show up if correct VCF files 201 formats are not selected, incorrect names of genomes were entered, or genome size was not entered 202 for calculating $p$ - or $J C$ distance. The pairwise diversity matrix could be copied and pasted on to 203 a text file, which could be used as an input for programs such as MEGA, PHYLIP or any other 204 software that accepts these formats. Hence users are able to use MEGA and other popular gene 205 specific software to edit or manipulate trees based on whole genome data. Similarly, the whole 206 genome based newick tree generated by VCF2PopTree could be further manipulated by the tree 207 editing software such as TreeGraph (Stover \& Muller 2010) or FigTree 208 (http://tree.bio.ed.ac.uk/software/figtree/).

\section{Conclusions}


211 VCF2PopTree is unique with respect to handling whole genome data from populations and it reads

212 data directly from the local machine and is independent of operating systems and browsers.

213 Importantly, this program does not require high performance computational resources, third party

214 software tools, a web server or internet connectivity. It is the fastest software available at present

215 to infer and draw population phylogeny in seconds to minutes. VCF2PopTree also produces

216 pairwise distant matrix and newick trees, which could be used as the input for the programs such

217 as MEGA or PHYLIP and thus facilitates whole genome based phylogenetic analysis through other

218 popular software. Therefore, VCF2PopTree could be a valuable phylogenetic tree building

219 software for researchers and students in the fields of Genetics, Ecology, Evolutionary Biology and

220 Medicine. VCF2PopTree is specifically developed to construct phylogenetic trees for whole

221 genome population data and not for that of species. Therefore, this software is not suited for

222 obtaining phylogenetic tree for species data.

\section{Funding}

225 This project was funded by a Linkage grant awarded to Sankar Subramanian by the Australian

226 Research Council (LP160100594).

\section{Acknowledgements}

229 The authors are thankful to all who tested the various versions of this software.

\section{Conflict of Interest}


232 None declared

233

234

235

236

237

238

239

240

241

242

243

244

245

246

247

248

249

250

251

252

\section{References}

Cook DE, and Andersen EC. 2017. VCF-kit: assorted utilities for the variant call format. Bioinformatics 33:1581-1582. 10.1093/bioinformatics/btx011

Dereeper A, Homa F, Andres G, Sempere G, Sarah G, Hueber Y, Dufayard JF, and Ruiz M. 2015. SNiPlay3: a web-based application for exploration and large scale analyses of genomic variations. Nucleic Acids Res 43:W295-300. 10.1093/nar/gkv351

Drummond AJ, Suchard MA, Xie D, and Rambaut A. 2012. Bayesian phylogenetics with BEAUti and the BEAST 1.7. Mol Biol Evol 29:1969-1973. 10.1093/molbev/mss075

Edgar RC. 2004. MUSCLE: multiple sequence alignment with high accuracy and high throughput. Nucleic Acids Res 32:1792-1797. 10.1093/nar/gkh340

Felsenstein J. 2005. Phylogeny Inference Package (Version 3.2). Cladistics 5:164-166.

Kaas RS, Leekitcharoenphon P, Aarestrup FM, and Lund O. 2014. Solving the problem of comparing whole bacterial genomes across different sequencing platforms. PLoS One 9:e104984. 10.1371/journal.pone.0104984

Kozlov AM, Aberer AJ, and Stamatakis A. 2015. ExaML version 3: a tool for phylogenomic analyses on supercomputers. Bioinformatics 31:2577-2579.

10.1093/bioinformatics/btv184

Kumar S, Stecher G, and Tamura K. 2016. MEGA7: Molecular Evolutionary Genetics Analysis Version 7.0 for Bigger Datasets. Mol Biol Evol 33:1870-1874. 10.1093/molbev/msw054 
253 Lee TH, Guo H, Wang X, Kim C, and Paterson AH. 2014. SNPhylo: a pipeline to construct a

254 phylogenetic tree from huge SNP data. BMC Genomics 15:162. 10.1186/1471-2164-15-

255

256

257

258

259

260

261

262

263

264

265

266

267

268

269

270

271

272

273

274

275 162

Lefort V, Desper R, and Gascuel O. 2015. FastME 2.0: A Comprehensive, Accurate, and Fast Distance-Based Phylogeny Inference Program. Mol Biol Evol 32:2798-2800. $10.1093 / \mathrm{molbev} / \mathrm{msv} 150$

Li H, and Durbin R. 2009. Fast and accurate short read alignment with Burrows-Wheeler transform. Bioinformatics 25:1754-1760. 10.1093/bioinformatics/btp324

Li H, Handsaker B, Wysoker A, Fennell T, Ruan J, Homer N, Marth G, Abecasis G, Durbin R, and Genome Project Data Processing S. 2009. The Sequence Alignment/Map format and SAMtools. Bioinformatics 25:2078-2079. 10.1093/bioinformatics/btp352

Liu L, Yu L, and Edwards SV. 2010. A maximum pseudo-likelihood approach for estimating species trees under the coalescent model. BMC Evol Biol 10:302. 10.1186/1471-2148-10302

Mallick S, Li H, Lipson M, Mathieson I, Gymrek M, Racimo F, Zhao M, Chennagiri N, Nordenfelt S, Tandon A, Skoglund P, Lazaridis I, Sankararaman S, Fu Q, Rohland N, Renaud G, Erlich Y, Willems T, Gallo C, Spence JP, Song YS, Poletti G, Balloux F, van Driem G, de Knijff P, Romero IG, Jha AR, Behar DM, Bravi CM, Capelli C, Hervig T, Moreno-Estrada A, Posukh OL, Balanovska E, Balanovsky O, Karachanak-Yankova S, Sahakyan H, Toncheva D, Yepiskoposyan L, Tyler-Smith C, Xue Y, Abdullah MS, RuizLinares A, Beall CM, Di Rienzo A, Jeong C, Starikovskaya EB, Metspalu E, Parik J, Villems R, Henn BM, Hodoglugil U, Mahley R, Sajantila A, Stamatoyannopoulos G, Wee JT, Khusainova R, Khusnutdinova E, Litvinov S, Ayodo G, Comas D, Hammer MF, 
276

277

278

279

280

281

282

283

284

285

286

287

288

289

290

291

292

293

294

295

296

297

298

Kivisild T, Klitz W, Winkler CA, Labuda D, Bamshad M, Jorde LB, Tishkoff SA, Watkins WS, Metspalu M, Dryomov S, Sukernik R, Singh L, Thangaraj K, Paabo S, Kelso J, Patterson N, and Reich D. 2016. The Simons Genome Diversity Project: 300 genomes from 142 diverse populations. Nature 538:201-206. 10.1038/nature18964

Patterson N, Moorjani P, Luo Y, Mallick S, Rohland N, Zhan Y, Genschoreck T, Webster T, and Reich D. 2012. Ancient admixture in human history. Genetics 192:1065-1093. 10.1534/genetics.112.145037

Pickrell JK, and Pritchard JK. 2012. Inference of population splits and mixtures from genomewide allele frequency data. PLoS Genet 8:e1002967. 10.1371/journal.pgen.1002967

Saitou N, and Nei M. 1987. The neighbor-joining method: a new method for reconstructing phylogenetic trees. Mol Biol Evol 4:406-425. 10.1093/oxfordjournals.molbev.a040454

Sokal R, and Michener C. 1958. A statistical method for evaluating systematic relationships. University of Kansas Science Bulletin 38:1409-1438.

Stamatakis A. 2006. RAxML-VI-HPC: maximum likelihood-based phylogenetic analyses with thousands of taxa and mixed models. Bioinformatics 22:2688-2690. 10.1093/bioinformatics/bt1446

Stover BC, and Muller KF. 2010. TreeGraph 2: combining and visualizing evidence from different phylogenetic analyses. BMC Bioinformatics 11:7. 10.1186/1471-2105-11-7

Vieiran FG, Lassalle F, Korneliussen TS, and Fumagalli M. 2016. Improving the estimation of genetic distances from Next-Generation Sequencing data. Biological Journal of the Linnean Society 117:139-149.

Wilgenbusch JC, and Swofford D. 2003. Inferring evolutionary trees with PAUP*. Curr Protoc Bioinformatics Chapter 6:Unit 6 4. 10.1002/0471250953.bi0604s00 
299 Xu D, Jaber Y, Pavlidis P, and Gokcumen O. 2017. VCFtoTree: a user-friendly tool to construct 300 locus-specific alignments and phylogenies from thousands of anthropologically relevant 301 genome sequences. BMC Bioinformatics 18:426. 10.1186/s12859-017-1844-0

302 
Figure 1

VCF2PopTree output on a web browser.

Screen shot of VCF2PopTree on the Google Chrome browser. (A) Input section (B) UPGMA Tree (C) Pairwise divergences in MEGA format and (D) Newick tree 


\section{VCF2PopTree}

Read VCF file

- Compressed VCF file (vcf.gz)

VCF file (.vcf)

Filter Single Nucleotide Variants (SNVs) by:

Minimum quality score

Minimum coverage depth
Enter score, depth

A

and choose input file

\begin{tabular}{lll} 
& Choose File & No file chosen \\
\hline Distance & CLEAR \\
\hline
\end{tabular}

Missing Data Use SNVs for each pair of genomes Use SNVs present in all genomes

Model $\quad$ Number of differences $\bigcirc$ P-distance $\quad$ Jukes-Cantor distance

Construct Tree $\quad \odot$ UPGMA tree $\bigcirc$ Neighbour-Joining tree (Unrooted)

Drawing options $\quad$ Rectangular tree $\bigcirc$ Radial tree

Output format $\bigcirc$ Newick tree $\bigcirc$ Pair-wise diversity (MEGA) $\bigcirc$ PHYLIP

Select genomes $\odot$ All $\bigcirc$ Selected

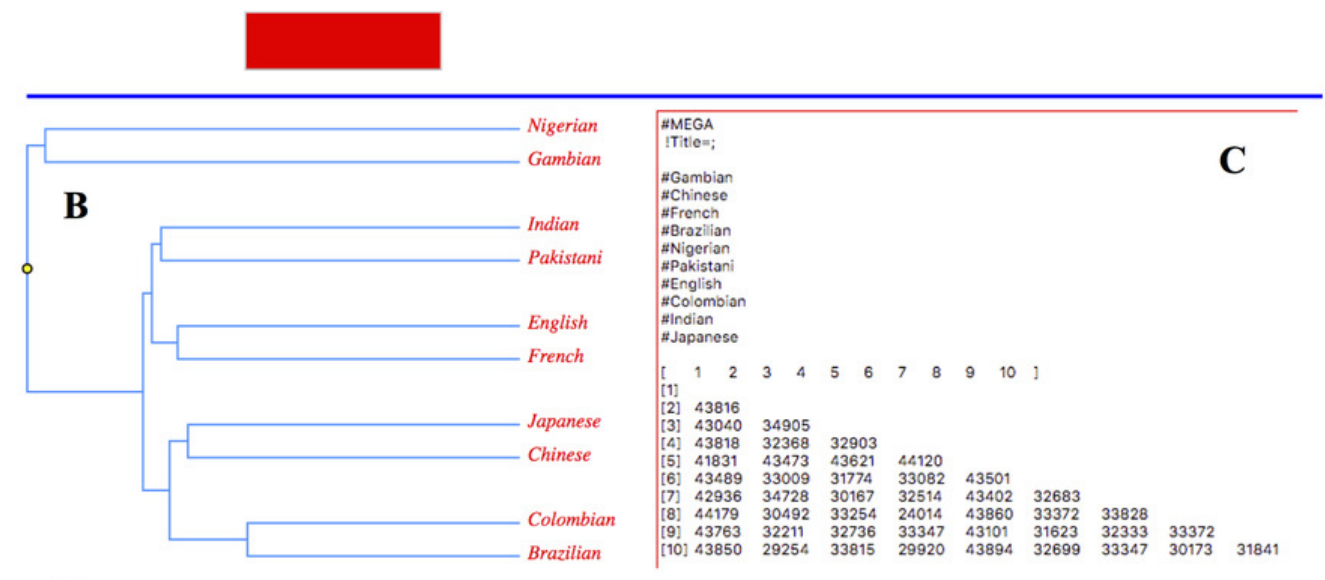

D

((Gambian:20915.5,Nigerian:20915.5):791.4820987654311,(((Pakistani:15811.25, Indian:15811.25):404.79166666666606, (French:15083.25,English:15083.25):1132.791666666666):390.84259259259306,((Chinese:14626.75, Japanese

:14626.75):809.9166666666679,(Brazilian:12007,Colombian:12007):3429.666666666668):1170.2175925925912):5100.097839506172); 
Figure 2

Comparison of VCF2PopTree with the popular online software, Galaxy.

(A) Correlation between the number of SNPs (or sites) and execution times of VCF2PopTree and Galaxy (B) Relationship between the number of genomes and execution times. Linear curves best fit the data points. Both correlations are highly significant $\left(r>0.98, P<10^{-6}\right)$ 

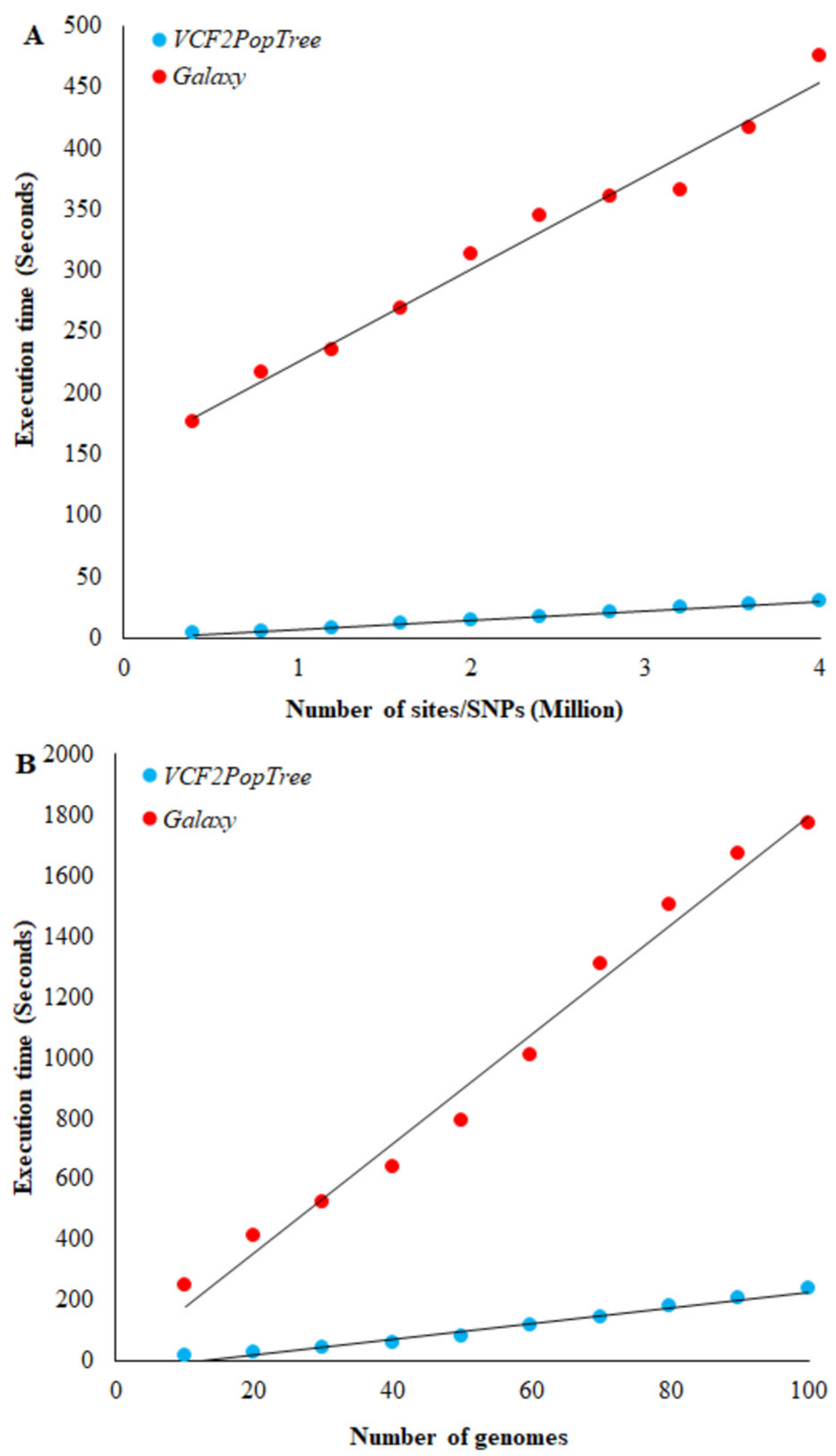

Peer) reviewing PDF | (2019:04:37132:2:0:NEW 4 Nov 2019) 